\title{
Protein Binding and Hepatic Clearance: Re-Examining the Discrimination between Models of Hepatic Clearance with Diazepam in the Isolated Perfused Rat Liver Preparation
}

\author{
Hong-Jaan Wang and Leslie Z. Benet \\ School of Pharmacy, National Defense Medical Center, Taipei, Taiwan (H.-J.W.), and Department of Bioengineering and \\ Therapeutic Sciences, Schools of Pharmacy and Medicine, University of California San Francisco, San Francisco, California (L.Z.B.) \\ Received July 29, 2019; accepted September 18, 2019
}

\begin{abstract}
This study re-examined the hepatic extraction for diazepam, the only drug for which isolated perfused rat liver (IPRL) studies have been reported not to be consistent with the well stirred model of organ elimination when only entering and exiting liver concentration measurements are available. First, the time dependency of diazepam equilibrium fraction unbound measurements from 4 to 24 hours was tested, reporting the continuing increases with time. The results showed that the time dependency of equilibrium protein-binding measurements for very highly bound drugs may be an issue that is not readily overcome. When examining $C_{\text {out }} / C_{\text {in }}\left(F_{\text {obs }}\right)$ measurements for diazepam when no protein is added to the incubation media, IPRL outcomes were consistent with previous reports showing marked underpredictability of in vivo clearance from in vitro measures of elimination in the absence of protein for very highly bound drugs, which is markedly diminished in the presence of albumin. $F_{\text {obs }}$ for diazepam at additional low concentrations of protein that would allow discrimination of the models of hepatic elimination produced results that were not consistent with the dispersion and
\end{abstract}

parallel-tube models. Therefore, although the outcomes of this study were similar to those reported by Rowland and co-workers, when no protein is added to the perfusion media, these IPRL results for diazepam cannot be reasonably interpreted as proving that hepatic organ elimination is model-independent or as supporting the dispersion and parallel-tube models of organ elimination.

\section{SIGNIFICANCE STATEMENT}

The only drug experiments for which isolated perfusion rat liver studies do not support hepatic clearance being best described by the well stirred model have been carried out with diazepam at zero protein concentration. This study repeated those studies, confirming the previous results at zero protein concentration, but the addition of low protein-binding conditions capable of differentiating the various models of hepatic elimination are more consistent with the well stirred model of hepatic elimination. These experimental studies do not support the preference for alternate models of hepatic elimination or the proposal that hepatic organ clearance is model-independent.

\section{Introduction}

In 2018, Benet et al. (2018) proposed that calculating organ clearance $(C L)$ as the product of the extraction ratio $(E R)$ and the blood flow to the organ $(Q)$ as given in eq. 1, as first proposed by Rowland (1972), was consistent with the well stirred model in pharmacokinetics. They maintain that this is true since the driving force concentration for elimination is assumed to be the concentration entering the organ, $C_{i n}$, rather than the concentration within the organ: Rate Out $=Q \cdot\left(C_{\text {in }}-C_{\text {out }}\right)=C L \cdot C_{i n}$. That is, there is no incremental clearance within the organ, and all elimination is driven only by the entering concentration:

$$
C L=Q \cdot E R=Q \cdot \frac{C_{\text {in }}-C_{\text {out }}}{C_{\text {in }}}
$$

This study was supported by the University of California San Francisco (UCSF) Benet Fund for Excellence generated from individual contributions and Dr. L.Z.B.'s consultation, expert witness, and board of director fees that are made payable to the Regents of the University of California. Dr. L.Z.B. is a member of the UCSF Liver Center supported by National Institutes of Health Grant P30 DK026743.

https://doi.org/10.1124/dmd.119.088872.
Rowland and Pang (2018) questioned this concept and have argued that eq. 1 "simply express[es] [a] proportionality between the observed rate of elimination and a reference concentration" and is not modeldependent. That is, clearance as calculated by eq. 1 is the organ clearance for all models of organ elimination, whether drug elimination in the organ follows the parallel-tube model, various axial dispersion models, or the well stirred model.

It is universally agreed that the various models of organ clearance can be differentiated only for high-clearance (ER) substrates, and in only three published studies is the difference in models for high ER drugs in rat isolated perfused organs directly tested. All three of those studies, including two from the Rowland laboratory, conclude that the data are consistent with the well stirred model, not alternate hepatic clearance models. Pang and Rowland (1977) evaluated the effect of changing organ blood flow on the ER of lidocaine; the Rowland laboratory also evaluated the effect of changing blood flow on the ER of meperidine (Ahmad et al., 1983); and Jones et al. (1984) evaluated the effect of changing protein binding on the extraction ratio of propranolol. Yet, these experimental results are generally ignored.

ABBREVIATIONS: $C_{\text {in }}$, entering concentration; $C L$, clearance; $\mathrm{CL}_{\text {int }}$, intrinsic clearance; $C_{\text {out }}$, exiting concentration; DZP, diazepam; ER, extraction ratio; $F_{\text {est }}$, estimated availability; $F_{\mathrm{obs}}$, observed availability; $f_{\mathrm{u}, \mathrm{B}}$, fraction unbound in blood; HSA, human serum albumin; IPRL, isolated perfusate rat liver; LC-MS/MS, liquid chromatography-tandem mass; $Q$, organ blood flow; RED, rapid equilibrium dialysis. 
In each of those studies for high-clearance (ER) drugs, the investigators concluded that the outcomes were consistent with the well stirred model as given in eq. 1 , as represented by eq. 2 , as opposed to the parallel tube model given in eq. 3 :

$$
\begin{gathered}
C L_{H}=\frac{Q_{H} \cdot f_{u, B} \cdot C L_{i n t, u}}{Q_{H}+f_{u, B} \cdot C L_{i n t, u}} \\
C L_{H}=Q_{H}\left(1-e^{\frac{-f_{u, B} \cdot C L_{i n t, u}}{\ell_{H}}}\right),
\end{gathered}
$$

where the hepatic clearance, $C L_{\mathrm{H}}$, is calculated from the hepatic blood flow, $Q_{\mathrm{H}}$, the fraction unbound in blood, $f_{\mathrm{u}, \mathrm{B}}$, and the unbound intrinsic clearance, $C L_{i n t, u}$.

Two experimental organ clearance studies with the low hepatic clearance drug, diazepam, in which diazepam has been manipulated to be high clearance in the absence of plasma proteins, were reported by the Rowland laboratory (Rowland et al., 1984; Díaz-García et al., 1992) to demonstrate preference for the parallel-tube and axial dispersion models versus the well stirred model. Yet, the only experimental data values detailed in these two articles showing preference for the alternate models were carried out when no protein was present in the perfusion media, and the outcomes $C_{\text {out }} / C_{\text {in }}$ measures differed by about 4 -fold: 0.042 $(\mathrm{CV}=76 \%)$ as reported by Rowland et al. (1984) using a gas liquid chromatographic analytical method and $0.011(\mathrm{CV}=45 \%)$ and reported by Díaz-García et al. (1992) using radiolabel measurements. Although the authors reported that "diazepam did not bind to the perfusion apparatus" (Díaz-García et al., 1992), we and other drug metabolism scientists are quite wary of carrying out studies in the absence of any binding protein in the drug containing media, especially since diazepam in human plasma is so highly protein bound (98.5\%; Allen and Greenblatt, 1980). There is also marked uncertainty as to how proteinbinding measurements should be interpreted and used in metabolism/ transport studies as we and others have reviewed (Poulin et al., 2016; Bowman and Benet, 2018; Bteich et al., 2019). In vitro measures in the absence of protein (albumin) markedly underpredict in vivo clearance; this difference is diminished in the presence of albumin (Poulin and Haddad, 2018; Kim et al., 2019). Therefore, in the present work, we have re-examined the rat liver perfusion clearance of diazepam in the absence of protein, but also at additional protein concentrations that discriminate, using mass spectrometric assay methods, which hepatic disposition model describes the hepatic clearance of diazepam.

\section{Materials and Methods}

Chemicals. With approval to use a controlled substance from the University of California San Francisco (UCSF), diazepam (DZP) and its internal standard diazepam-d5 were obtained from Spectrum (Gardena, CA) and Cerilliant (Round Rock, TX), respectively. The buffer reagents used in the IPRL experiments, including taurocholate, sodium bicarbonate, and Krebs-Ringer powder, were all purchased from Sigma-Aldrich (St. Louis, MO). Fatty acid-free human serum albumin (HSA) was supplied commercially from SeraCare (Milford, MA). Rapid equilibrium dialysis (RED) device inserts were purchased from Thermo Scientific (Rockford, IL). All other chemicals and solvents for the analysis were of analytical or liquid chromatography-mass spectrometry (LC-MS) grade.

Diazepam Protein Binding. Equilibrium dialysis experiments with DZP were carried out using RED device inserts (Waters et al., 2008). DZP was prepared at $1 \mu \mathrm{g} / \mathrm{ml}$ in Krebs' buffer containing varied HSA concentrations (w/v) of $0 \%, 0.025 \%, 0.05 \%, 0.1 \%, 0.5 \%, 1 \%, 2 \%$, and $4 \%$. A $500-\mu 1$ volume of DZP testing solution was spiked into the sample chamber of the insert, followed by the addition of $750 \mu \mathrm{l}$ of blank buffer in the adjacent chamber. The loaded inserts were assembled in the Teflon-based plate, and the plate was sealed with a self-adhesive lid, swayed at 20 strokes per minute under $37^{\circ} \mathrm{C}$ on an incubator shaker (Feasterville, PA) for the required experimental times of 4, 8, 12, or 24 hours.
Surgery and Perfusion of Livers. Male Sprague-Dawley rats (350-400 g; Charles River Laboratory, Hollister, CA) were housed in the UCSF animal care facility with a 12-hour light/dark cycle and allowed free access to water and food. Approval for their use in experiments was obtained from the Committee on Animal Research of UCSF. Anesthesia was achieved by intraperitoneal injection with a $1 \mathrm{ml} / \mathrm{kg}$ dose of ketamine/xylazine $(91 \mathrm{mg}, 9.1 \mathrm{mg} / \mathrm{ml}$ ) before surgery. Livers were isolated for perfusion ex situ, as described previously with slight modifications (Wu and Benet, 2003; Lau et al., 2004). Oxygenated Krebs-Ringer bicarbonate buffer ( $\mathrm{pH}$ 7.4), supplemented with different concentrations of HSA (ranging from $0 \%$ to $2 \%$ ), sodium bicarbonate $(15 \mathrm{mM}$ ), and sodium taurocholate $(10 \mu \mathrm{M})$, was pumped through the liver at a flow rate of $15 \mathrm{ml} / \mathrm{min}$ via a catheter inserted in the portal vein. Perfusion was performed in eight rats at $37^{\circ} \mathrm{C}$ in a single-pass manner from a reservoir containing $500 \mathrm{ml}$ of medium, through a $1-\mu \mathrm{m}$ pore-size glass fiber filter, oxygenator, and bubble trap placed before the liver. The perfusate was oxygenated by carbogen $\left(95 \% \mathrm{O}_{2} / 5 \% \mathrm{CO}_{2}\right)$ as it passed through the semipermeable tube before entering the liver. Liver viability was judged on the basis of its appearance (uniformly pink to brown), portal vein pressure (8-10 $\mathrm{mm} \mathrm{Hg}$ ), and the $\mathrm{pH}$ of perfusate (in the range of 7.35-7.45), as well as metabolic capability. After a 20-minute stabilization period, single-pass perfusions were started sequentially, with the perfusates containing $1 \mu \mathrm{g} / \mathrm{ml}$ DZP and different HSA concentrations $(0 \%, 0.025 \%, 0.05 \%, 0.1 \%, 0.5 \%$, and $2.0 \%)$. The order of the perfusate concentrations randomly followed one of the two sequences depicted in Fig. 1. During each rat perfusion experiment, 2-ml samples from the inferior vena cava $\left(C_{\text {out }}\right)$ were taken at $16,17,18,19$, and 20 minutes. At the end of each perfusion period, a 2-ml influx sample $\left(C_{i n}\right)$ was obtained. Because of fluctuating bile flow rate from the cannulated bile duct after liver isolation, no attempt was made to quantitate DZP in the bile.

Sample Preparation and Analysis. A simple, quick method of protein precipitation was used in sample preparation. Each $200-\mu 1$ perfusate sample was combined with $40 \mu \mathrm{l}$ of internal standard solution ( $500 \mathrm{ng} / \mathrm{ml}$ in acetonitrile), as well as $560 \mu 1$ of cold acetonitrile. The final sample/internal standard/organic solvent mixtures were then mixed briefly, followed by centrifugation at $13,000 \mathrm{~g}$ for 10 minutes. Then, $200 \mu \mathrm{l}$ of the supernatant was transferred into highperformance liquid chromatography screw-cap vials. Preliminary studies indicated no matrix effects as a function of different HSA concentrations; therefore, calibration curves for DZP were constructed over the range of $1-1000 \mathrm{ng} / \mathrm{ml}$ in perfusate with $2 \%$ HSA.

DZP and its internal standard DZP-d5 were analyzed using a Shimadzu LC-20AD high-performance liquid chromatography (Kyoto, Japan) coupled to a Biosystems-Sciex API 4000 series triple-quadrupole mass spectrometer (Foster City, CA) with an electrospray ionization interface. Chromatographic separation was carried out on a Thermo BDS Hypersil C18 column $(4.6 \times 100 \mathrm{~mm}, 5 \mu \mathrm{m}$; Thermo Fisher, Waltham, MA) using a mobile phase composed of solvent A ( $2 \mathrm{mM}$ ammonium acetate and $0.1 \%$ formic acid in water) and solvent $\mathrm{B}(2 \mathrm{mM}$ ammonium acetate and $0.1 \%$ formic acid in methanol) under an isocratic program with a 3-minute run time and a $0.7 \mathrm{ml} / \mathrm{min}$ flow rate. The autosampler was conditioned at $10^{\circ} \mathrm{C}$, and the injection volume was set at $10 \mu \mathrm{l}$. Acquisition for

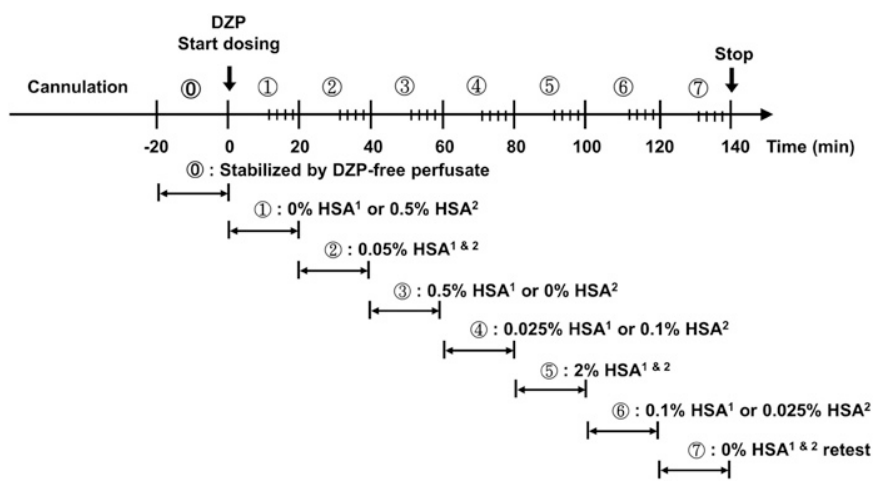

Fig. 1. Drug-loading design of diazepam $(1 \mu \mathrm{g} / \mathrm{ml})$ combined with varied HSA concentrations in IPRL studies. Two sequences of HSA concentrations were used, and individual rats were randomly assigned to a sequence. The Arabic number superscripts indicate where the individual HSA concentrations were in the testing sequence (i.e., in sequence 1, 0.1\% HSA was studied between 100 and 120 minutes, and in sequence 2, this HSA concentration was studied between 60 and 80 minutes). 
A

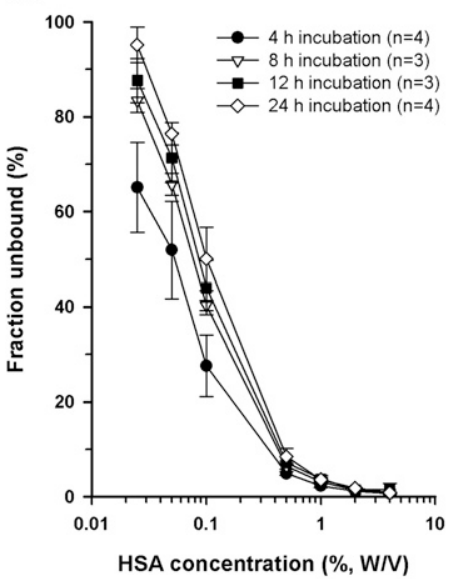

B

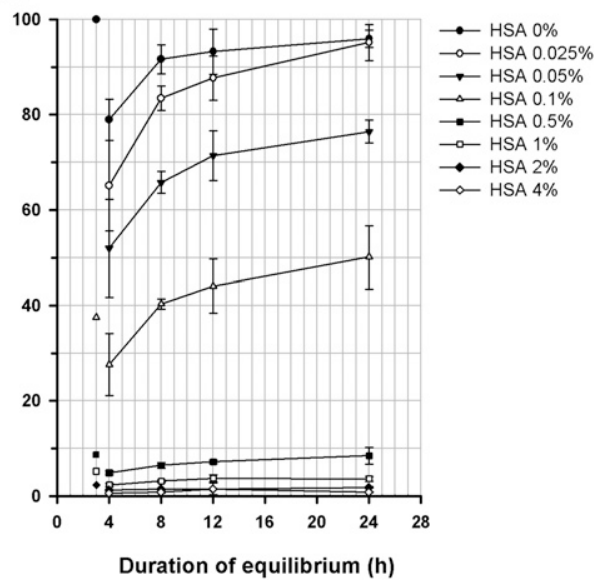

Fig. 2. (A) Plot of the free fraction of diazepam in the perfusate as a function of logarithmic HSA concentration for incubation times ranging from 4 to 24 hours. (B) Solid lines connect the fraction unbound as a function of equilibration time reported here. Data points at 3 hours represent the fraction unbound reported by DíazGarcía et al. (1992). tandem mass spectrometry was performed in positive ionization mode, and the multiple reaction monitoring mode was selected for quantification of the analytes. The precursors to production ion transitions in the analytes were $285.2 \rightarrow 154.1$ and $285.2 \rightarrow 193.0$ for DZP, as well as 290.2 $\rightarrow 154.1$ for DZP-d5 (Marin et al., 2012). The ion-source temperature was maintained at $550^{\circ} \mathrm{C}$, and the ion spray voltage was $5.0 \mathrm{kV}$. Analyst 1.4.2 software (Applied Biosystems-Sciex, Foster City, CA) was used to collect and process the data.

Models of Hepatic Drug Clearance. Three models of hepatic elimination are considered in the figures comparing the outcomes by Díaz-García et al. (1992): the well-stirred model, the parallel-tube model, and the axial dispersion model with a dispersion number of 0.34 . The theoretical figures comparing the various hepatic models with changing protein binding or changing flow rate are based on the assumption that eq. 1 is model independent. Although we disagree with that assumption, and that only the well-stirred model simulation is relevant, we calculated the theoretical curves here, following the procedure described in DíazGarcía et al. (1992), so that a comparison with previous publications can be made. This was done by using the mean observed $f_{\mathrm{u}}$ and $C_{\text {out }} / C_{\text {in }}\left(F_{\text {obs }}\right)$ values for all protein concentrations and estimating the $C L_{i n t, u}$ for each of the models. This value was then used to determine the estimated $C_{\text {out }} / C_{\text {in }}\left(F_{\text {est }}\right)$ values at each value of $f_{u}$, from which the hypothetical curve representing each of the models could be drawn.

\section{Results}

Equilibrium Dialysis. Variations of diazepam protein binding under different HSA concentrations are shown in Fig. 2 and Table 1, reflecting the time-dependent changes in diazepam protein binding as a function of equilibration times between 4 and 24 hours. The time dependency of

TABLE 1

Diazepam fraction unbound (\%) as a function of incubation time and human serum albumin (HSA) concentration

\begin{tabular}{|c|c|c|c|c|c|}
\hline Dialysis Time & HSA $(\% \mathrm{w} / \mathrm{v})$ & Mean & SD & $\mathrm{CV} \%$ & Ratio $24 \mathrm{~h} / 4 \mathrm{~h}$ \\
\hline \multirow{8}{*}{$4 \mathrm{~h}(n=4)$} & 0 & 78.9 & 4.3 & 5.5 & \\
\hline & 0.025 & 65.1 & 9.5 & 14.6 & \\
\hline & 0.05 & 52.0 & 10.2 & 19.6 & \\
\hline & 0.1 & 27.6 & 6.5 & 23.5 & \\
\hline & 0.5 & 4.91 & 0.29 & 8.0 & \\
\hline & 1 & 2.34 & 0.34 & 14.7 & \\
\hline & 2 & 1.25 & 0.11 & 8.8 & \\
\hline & 4 & 0.646 & 0.042 & 6.5 & \\
\hline \multirow[t]{8}{*}{$8 \mathrm{~h}(n=3)$} & 0 & 91.6 & 3.0 & 3.3 & \\
\hline & 0.025 & 83.4 & 2.5 & 3.0 & \\
\hline & 0.05 & 65.8 & 2.3 & 3.5 & \\
\hline & 0.1 & 40.3 & 1.1 & 2.6 & \\
\hline & 0.5 & 6.44 & 0.63 & 9.8 & \\
\hline & 1 & 3.17 & 0.26 & 8.3 & \\
\hline & 2 & 1.46 & 0.05 & 3.4 & \\
\hline & 4 & 0.826 & 0.201 & 24.3 & \\
\hline \multirow[t]{8}{*}{$12 \mathrm{~h}(n=3)$} & 0 & 93.2 & 4.8 & 5.1 & \\
\hline & 0.025 & 87.7 & 4.7 & 5.3 & \\
\hline & 0.05 & 71.4 & 5.2 & 7.3 & \\
\hline & 0.1 & 44.0 & 5.7 & 7.3 & \\
\hline & 0.5 & 7.18 & 0.39 & 5.5 & \\
\hline & 1 & 3.75 & 0.76 & 20.2 & \\
\hline & 2 & 1.52 & 0.08 & 5.1 & \\
\hline & 4 & 0.818 & 0.137 & 16.7 & \\
\hline \multirow[t]{8}{*}{$24 \mathrm{~h}(n=4)$} & 0 & 96.0 & 1.8 & 1.9 & 1.22 \\
\hline & 0.025 & 95.2 & 3.8 & 4.0 & 1.46 \\
\hline & 0.05 & 76.4 & 2.4 & 3.1 & 1.47 \\
\hline & 0.1 & 50.1 & 6.6 & 13.2 & 1.81 \\
\hline & 0.5 & 8.48 & 1.79 & 21.2 & 1.73 \\
\hline & 1 & 3.62 & 0.48 & 13.3 & 1.55 \\
\hline & 2 & 1.81 & 0.17 & 9.6 & 1.51 \\
\hline & 4 & 0.837 & 0.106 & 12.7 & 1.30 \\
\hline
\end{tabular}




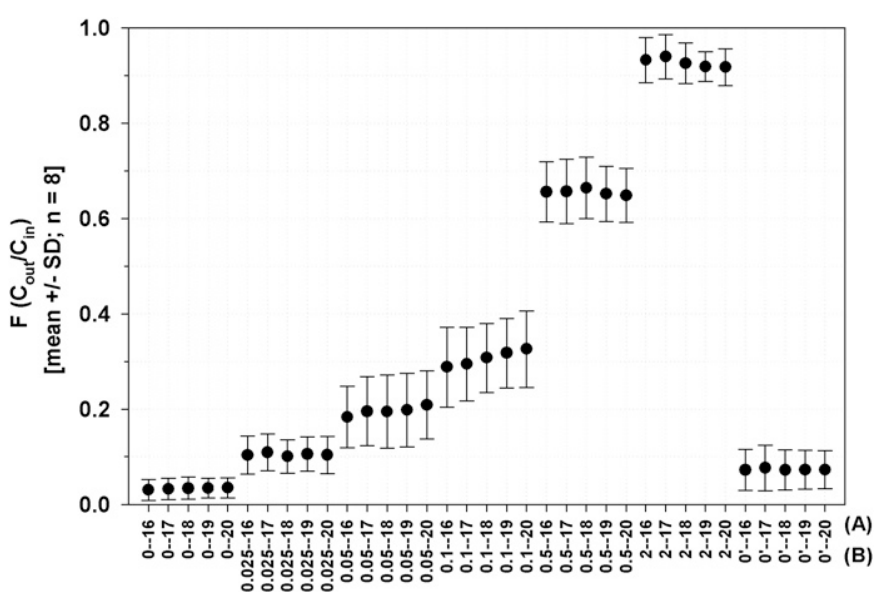

(A): Sampling time in each period $(\mathrm{min})$

(B): HSA concentration (\%)

Fig. 3. The availabilities of diazepam in IPRL studies under varied HSA concentrations in a total of seven periods with constant perfusate flow rate $(15 \mathrm{ml} / \mathrm{min})$ and drug concentration $(1 \mu \mathrm{g} / \mathrm{ml})$. Flow-out samples were collected every minute from 16 to 20 minutes in each period, including the flow-in perfusate at the last sampling time.

equilibrium protein-binding measurements is well recognized. For example, Obach (1997) demonstrated it to be a potential error source for in vitro-in vivo extrapolation predictions for warfarin, imipramine, and propranolol. Table 1 presents the mean values of percent fraction unbound for the four different dialysis times at the eight different HSA concentrations. The table also presents the standard deviations at each HSA concentration and the coefficient of variation of these measures. Although there are differences in variability (coefficient of variation) at different dialysis times and different HSA concentrations, no pattern is discernable. That is, the coefficients of variation appear to be randomly distributed across the 32 different mean values. Particularly relevant is comparison of the ratio of mean protein-binding values at each of the HSA concentrations for the 24-hour measurements compared with the 4-hour measurements. The change in the extent of binding for zero HSA concentration, where the fraction unbound percentages increase from $78.9 \%$ to $96.0 \%$ over the 18 -hour time period (ratio of 1.22 ), was very similar to the proportional change in the extent of protein binding at $4 \% \mathrm{HSA}$, where the percent unbound increased from $0.65 \%$ to $0.84 \%$ (ratio of 1.30). The protein-binding change for all HSA concentrations changed similarly, ranging from 1.22 to 1.82 . Thus, although it may appear in Fig. 2A that significant changes in protein binding with respect to incubation time occur only at low HSA concentration, the actual data as presented in Table 1 demonstrate that the relative change in the extent of binding from 4 to 24 hours is approximately the same for all HSA concentrations.

IPRL Experiments. We conducted IPRL using eight male rat livers using two different perfusion sequences and varying HSA concentrations (Fig. 1). Figure 3 depicts the observed $C_{\text {out }} / C_{\text {in }}$ ratios for all eight rats at the six different HSA concentrations, each with five measurements taken at minutes $16-20$, as indicated. Two perfusion sequences were used; therefore, $C_{\text {out }} / C_{\text {in }}$ ratios were averaged for the two sequences. The final $0 \%$ HSA measurement in each rat (depicted as 0 ') is shown separately from the average of the earlier 0\% HSA perfusions. Compared with the first period of HSA-free perfusion (0-20 or 40-60 minutes), the availability of DZP at 120-140 minutes was increased approximately 2-fold $(0.033 \% \pm 0.022 \%$ vs. $0.073 \% \pm$ $0.043 \%, P<0.05)$. The individual results for the eight rats in the IPRL studies are depicted in Fig. 4, where the $0 \%$ HSA results are for the initial

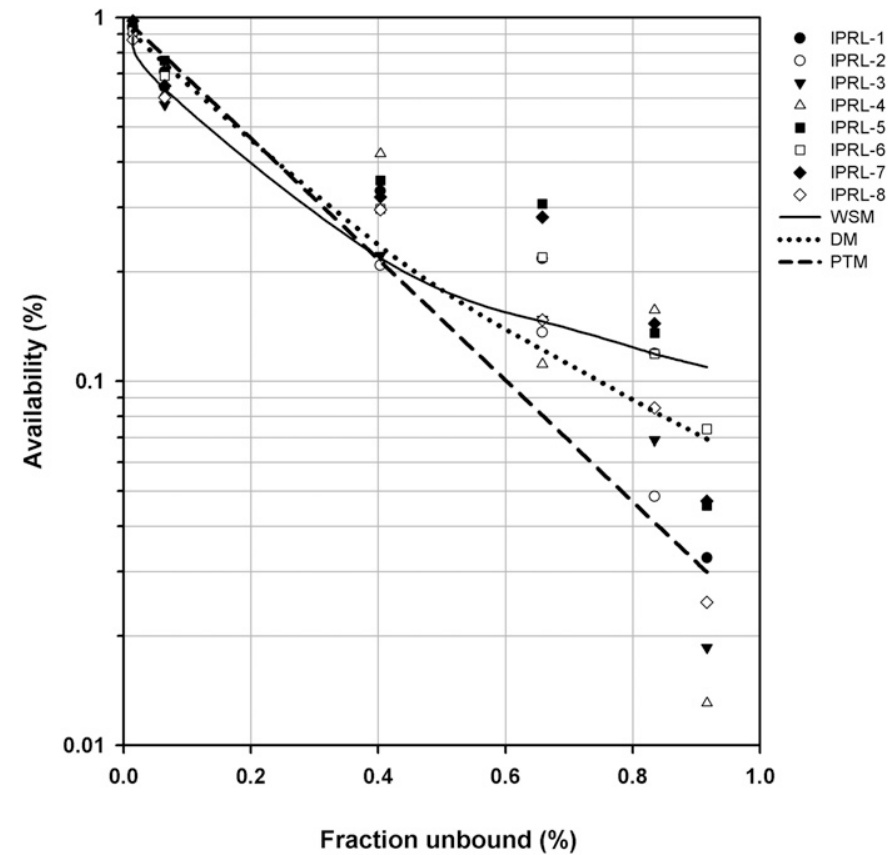

Fig. 4. Estimated results of well stirred (WSM), parallel-tube (PTM), and dispersion (DM) models for the relationship between availability and fraction unbound of diazepam. Symbols represent the individual results in each of the IPRL studies. Values for the zero protein added to the incubation are for the initial measurements.

measurements only. The average $F_{\text {obs }}$ values are presented in Table 2, together with the calculated $F_{\text {est }}$ for each model using the average 8-hour equilibrium fraction unbound measurements for each HSA concentration. (Although $F_{\text {est }}$ values would differ slightly for the different "equilibrium times" chosen, the differentiation between models would be similar.) The mean $F_{o b s}$ values were then used for each model to generate the theoretical curves, as depicted in Figs. 4 and 5. Figure 4 displays the individual data, and Fig. 5 depicts the average of the experimental $F_{o b s}$ values for each HSA concentration compared with fraction unbound and the theoretical curves for the three models of hepatic elimination. The last three columns of Table 2 give the estimated $F$ values for each model for each HSA concentration.

\section{Discussion}

The motivation for the present study resulted from our interest in reexamining the data from apparently the only isolated rat liver perfusion drug studies that provided results that were not best explained by the well stirred model of organ clearance. Those studies (Rowland et al., 1984; Díaz-García et al., 1992) examined diazepam clearance and protein binding at HSA concentrations of $0 \%, 0.1 \%, 0.5 \%, 1 \%$, and $2 \%$. The authors reported that "Preliminary experiments showed that equilibrium was reached within 3 hours, that volume shifts were negligible, and that diazepam did not bind to the experimental system." The mean experimental percent fraction unbound values from these studies are included in Fig. 2B and shown at 3 hours. Our study using LC-MS/MS measurements in the RED device yielded lower results.

The variation of diazepam protein binding under different HSA concentrations is shown in Fig. 2. The fraction unbound of diazepam in the HSA-free medium was $78.9 \%$ after 4-hour incubation, not reaching the theoretical value of $100 \%$ even after a 24-hour incubation, as opposed to the $100 \%$ free fraction reported by Díaz-García et al. (1992) after a 3-hour incubation. As shown in Table 1, the fractions unbound at $0.1 \%$ HSA ranged from $27.6 \%$ after 4 -hour incubation to $50.1 \%$ after 24-hour incubation compared with the reported $37.6 \%$ after a 3-hour 
TABLE 2

Observed $\left(F_{\text {obs }}\right)$ and estimated availability $\left(F_{\text {est }}\right)$ of diazepam in the human serum albumin under varying protein (HSA) binding conditions and measured 8-hour fraction unbound for three different hepatic drug clearance models: WSM, PTM, and DM.

\begin{tabular}{lcccrr}
\hline \multirow{2}{*}{ HSA $(\%)$} & $F_{\mathrm{ubs}, 8 \mathrm{~h}}(\%)$ & & \multicolumn{2}{c}{ Estimated Availability; $F_{\text {est }}(\%)$} \\
\cline { 4 - 6 } & & & WSM & PTM & DM \\
\hline 0 & 0.92 & 3.3 & 10.7 & 3.0 & 6.9 \\
0.025 & 0.83 & 10.9 & 11.7 & 4.1 & 8.1 \\
0.05 & 0.66 & 30.6 & 14.3 & 8.1 & 12.1 \\
0.5 & 0.40 & 65.6 & 21.4 & 21.3 & 23.3 \\
2 & 0.06 & 92.7 & 88.1 & 78.1 & 74.4 \\
\hline
\end{tabular}

DM, dispersion model; PTM, parallel-tube model; WSM, well stirred model.

incubation by Díaz-García et al. (1992). None of the protein binding measurements at various HSA concentrations reached equilibrium at 24 hours, all of which exhibit a similar degree of nonequilibrium, independent of protein concentration. Further, the variability of the measurement and the difference of the measurement between 4 and 24 hours appear to be independent of protein concentration. We conclude that the time dependency of equilibrium protein-binding measurements for very highly bound drugs may be an issue that is not readily overcome.

For the isolated perfused diazepam liver studies, we showed results similar to those of Rowland and co-workers for the initial measurements in the absence of added protein. That is, the mean data for this point $\left(0 \%\right.$ HSA; $91.6 \%$ average fraction unbound; $\left.F_{\text {obs }}=0.033\right)$ appear to be consistent with the parallel-tube model (see Figs. 4 and 5). As depicted in Fig. 4, there is great variability in the eight individual measurements at zero protein addition $\left(F_{o b s}=0.033 ; \mathrm{CV}=67 \%\right)$, with this high variability $\left(F_{o b s}=0.042 ; \mathrm{CV}=76 \%\right)$ also reported by Rowland et al. (1984) using a gas liquid chromatographic analytical method and $\left(F_{o b s}=0.011 ; \mathrm{CV}=45 \%\right)$ reported by Díaz-García et al. (1992) using radiolabel measurements. Our second measures at zero protein

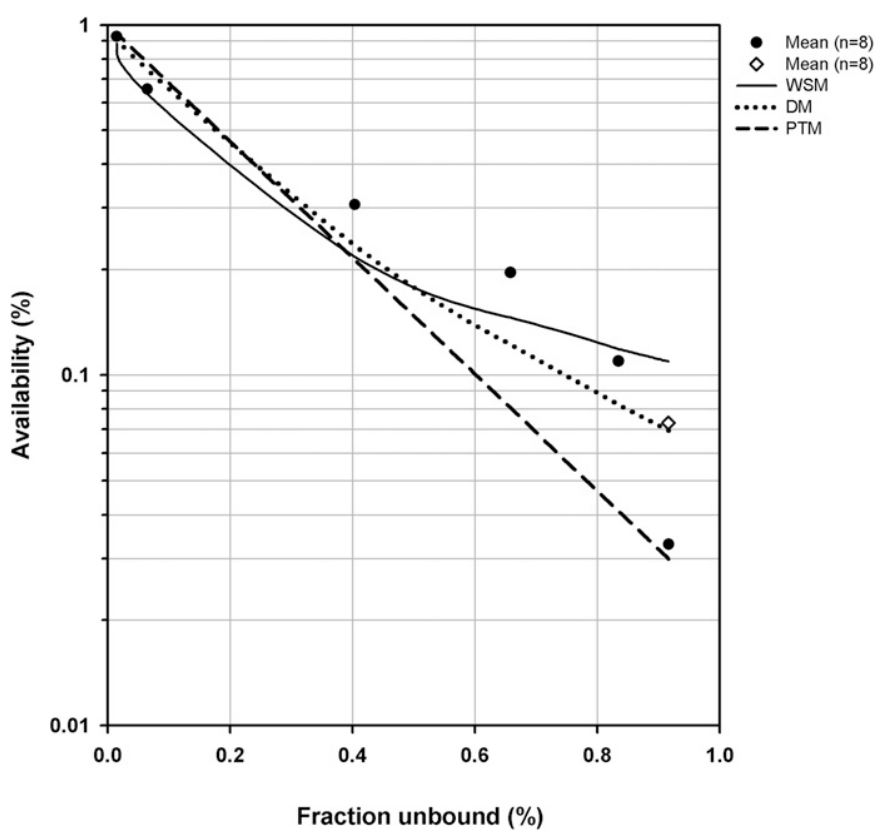

Fig. 5. Estimated results of well stirred (WSM), parallel-tube (PTM), and dispersion (DM) models for the relationship between availability and fraction unbound of diazepam. Symbols represent the mean results $(n=8)$ in all of the IPRL studies. For zero protein additions, -Initial measurements between 0 and 20 minutes and 40-60 minutes; $\diamond$ Second measurements in each perfusion at 120-140 minutes. addition of $F_{\text {obs }}$ at $120-140$ minutes $\left(F_{\text {obs }}=0.073 \% ; \mathrm{CV}=59 \%\right)$ were statistically higher than the earlier first measurements, probably reflecting the decrease in liver function with time, as this parameter is much more sensitive than traditional measures of liver viability. When our second measure at zero protein was plotted in Fig. 5, this mean value appears to be more consistent with the dispersion model (0\% HSA; $91.6 \%$ average fraction unbound; $\left.F_{o b s}=0.073\right)$. The 2 -fold difference here in our measurements should be considered in light of the 4-fold difference reported in the two studies by Rowland and co-worker.

The investigations of Rowland and co-workers did not study any other protein concentrations that would readily allow comparison of the different models. Thus, we investigated $0.025 \%$ and $0.05 \%$ protein concentrations in our IPRL studies. As seen in Figs. 4 and 5, these studies appear to be more consistent with the well stirred model. Table 2 shows the estimated availability for each of the models (under the assumption that clearance was model-independent). At lower protein concentrations, where the diazepam clearance (ER) is high, differentiation between the models is obvious. This differentiation dissipates as protein binding increases and clearance (ER) decreases. Comparing the least-square differences between the three models as presented in Table 2 and shown in Fig. 5, the initial average $F_{o b s}$ measurement when no protein is added to the perfusion media is consistent with the theoretical parallel-tube curve and the later 120- to 140-minute measurement is consistent with the dispersion model theoretical curve. Thus, it appears that we confirm the experimental results observed by Rowland et al. (1984) and Diaz-Garcia et al. (1992), respectively, when no protein is added to the perfusion media; however, when we studied low concentrations of protein that could differentiate the various theoretical models, our results appear to be consistent with the well stirred model. As noted, currently there is increasing attention being paid to how fraction unbound is interpreted in predicting metabolic interactions and the effect that albumin can have on these protein-binding and metabolism measurement as measured in the absence in protein markedly underpredict in vivo clearance as recently reviewed (Bowman and Benet, 2018; Bteich et al., 2019). Recent studies with hepatocytes report markedly better in vitro-in vivo extrapolation predictability in the presence of albumin than in its absence (Poulin and Haddad, 2018; Kim et al., 2019). Thus, although we can confirm similar outcomes to that reported by Rowland and co-workers when no protein is added to perfusion media, those results cannot be reasonably interpreted as proving that eq. 1 is model-independent.

\section{Authorship Contributions}

Participated in research design: Wang, Benet.

Conducted experiments: Wang.

Performed data analysis: Wang, Benet.

Wrote or contributed to the writing of the manuscript: Wang, Benet. 


\section{References}

Ahmad AB, Bennett PN, and Rowland M (1983) Models of hepatic drug clearance: discrimination between the 'well stirred' and 'parallel-tube' models. J Pharm Pharmacol 35:219-224.

Allen MD and Greenblatt DJ (1980) Comparative protein binding of diazepam and desmethyldiazepam. J Clin Pharmacol 20:639-643.

Benet LZ, Liu S, and Wolfe AR (2018) The universally unrecognized assumption in predicting drug clearance and organ extraction ratio. Clin Pharmacol Ther 103:521-525.

Bowman CM and Benet LZ (2018) An examination of protein binding and protein-facilitated uptake relating to in vitro-in vivo extrapolation. Eur J Pharm Sci 123:502-514.

Bteich M, Poulin P, and Haddad S (2019) The potential protein-mediated hepatic uptake: discussion on the molecular interactions between albumin and the hepatocyte cell surface and their implications for the in vitro-to-in vivo extrapolations of hepatic clearance of drugs. Expert Opin Drug Metab Toxicol 15:633-658.

Díaz-García JM, Evans AM, and Rowland M (1992) Application of the axial dispersion model of hepatic drug elimination to the kinetics of diazepam in the isolated perfused rat liver. J Pharmacokinet Biopharm 20:171-193.

Jones DB, Morgan DJ, Mihaly GW, Webster LK, and Smallwood RA (1984) Discrimination between the venous equilibrium and sinusoidal models of hepatic drug elimination in the isolated perfused rat liver by perturbation of propranolol protein binding. J Pharmacol Exp Ther 229: $522-526$.

Kim SJ, Lee KR, Miyauchi S, and Sugiyama Y (2019) Extrapolation of in vivo hepatic clearance from in vitro uptake clearance by suspended human hepatocytes for anionic drugs with high binding to human albumin: improvement of in vitro-to-in vivo extrapolation by considering the "albumin-mediated" hepatic uptake mechanism on the basis of the "facilitated-dissociation model". Drug Metab Dispos 47:94-103.

Lau YY, Wu C-Y, Okochi H, and Benet LZ (2004) Ex situ inhibition of hepatic uptake and efflux significantly changes metabolism: hepatic enzyme-transporter interplay. J Pharmacol Exp Ther 308: $1040-1045$.

Marin SJ, Roberts M, Wood M, and McMillin GA (2012) Sensitive UPLC-MS-MS assay for 21 benzodiazepine drugs and metabolites, zolpidem and zopiclone in serum or plasma. $J$ Anal Toxicol 36:472-476.
Obach RS (1997) Nonspecific binding to microsomes: impact on scale-up of in vitro intrinsic clearance to hepatic clearance as assessed through examination of warfarin, imipramine, and propranolol. Drug Metab Dispos 25:1359-1369.

Pang KS and Rowland M (1977) Hepatic clearance of drugs. II. Experimental evidence for acceptance of the "well-stirred" model over the "parallel tube" model using lidocaine in the perfused rat liver in situ preparation. $J$ Pharmacokinet Biopharm 5:655-680.

Poulin P, Burczynski FJ, and Haddad S (2016) The role of extracellular binding proteins in the cellular uptake of drugs: impact on quantitative in vitro-to-in vivo extrapolations of toxicity and efficacy in physiologically based pharmacokinetic-pharmacodynamic research. J Pharm Sci 105:497-508

Poulin P and Haddad S (2018) Extrapolation of the hepatic clearance of drugs in the absence of albumin in vitro to that in the presence of albumin in vivo: comparative assessement of 2 extrapolation models based on the albumin-mediated hepatic uptake theory and limitations and mechanistic insights. J Pharm Sci 107:1791-1797.

Rowland M (1972) Influence of route of administration on drug availability. J Pharm Sci 61:70-74.

Rowland M, Leitch D, Fleming G, and Smith B (1984) Protein binding and hepatic clearance: discrimination between models of hepatic clearance with diazepam, a drug of high intrinsic clearance, in the isolated perfused rat liver preparation. J Pharmacokinet Biopharm 12:129-147.

Rowland M and Pang KS (2018) Commentary on "The universally unrecognized assumption in predicting drug clearance and organ extraction ratio". Clin Pharmacol Ther 103:386-388.

Waters NJ, Jones R, Williams G, and Sohal B (2008) Validation of a rapid equilibrium dialysis approach for the measurement of plasma protein binding. J Pharm Sci 97:4586-4595.

Wu C-Y and Benet LZ (2003) Disposition of tacrolimus in isolated perfused rat liver: influence of troleandomycin, cyclosporine, and GG918. Drug Metab Dispos 31:1292-1295.

Address correspondence to: Dr. Leslie Z. Benet, Department of Bioengineering and Therapeutic Sciences, Schools of Pharmacy and Medicine, University of California San Francisco, 533 Parnassus Avenue, Room U-68, San Francisco, CA 94143-0912. E-mail: leslie.benet@ucsf.edu 